\title{
Calcificação pulpar interna em dente de paciente idoso: relato de caso
}

\author{
Internal pulp calcification in an elderly patient's tooth: case report
}

Calcificación pulpar interna en el diente de un paciente anciano: report de un caso

Evalda Santana Reis Santiago ${ }^{1 *}$, Tatiane Mandu Silva ${ }^{1}$, Sandra Stephanie da Silva Rosales ${ }^{1}$, Clelia Alice Tourinho de Melo e Silva ${ }^{1}$, Maria Luiza Paes Barreto dos Santos Batista ${ }^{1}$, Ana Carolina Reis

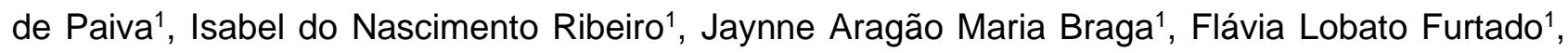
Douglas Oliveira de Souza 1.

\section{RESUMO}

Objetivo: $O$ objetivo deste trabalho é apresentar um relato de caso clínico em um paciente idoso atendido na clínica Odontogeriátrica em uma Universidade no estado do Pará na cidade de Belém, o qual apresentava calcificação interna do dente 47. Detalhamento do caso: Trata-se de um caso de calcificação pulpar causada pelo processo de envelhecimento, comprovado por radiografia periapical: paciente de 70 anos, gênero masculino, o qual apresentava o dente $47 \mathrm{com}$ uma restauração metálica fundida extensa mesio-oclusal, e fistula vestibular à altura da bifurcação das raízes. Foram feitos tratamentos endodônticos no filete radicular da raiz distal, curetagem da fistula sob anestesia local, restauração provisória e prescrição medicamentosa de acordo com a necessidade clínica do paciente. Considerações finais: Podemos concluir sobre o relato que há uma necessidade de os cirurgiões dentistas buscarem maior conhecimento sobre alterações bucais causadas pelo envelhecimento, para que possam por meio de diagnóstico comprovado indicar tratamentos mais conservadores em dentes calcificados.

Palavras-chave: Calcificação, Envelhecimento, Tratamento.

\begin{abstract}
Objective: The objective of this study is to present a clinical case report in an elderly patient treated at the Odontogeriatric clinic at a University in the state of Pará in the city of Belém, which had internal calcification of tooth 47. Details of the case: This is a case of pulp calcification caused by the aging process, confirmed by periapical radiography: 70 -year-old male patient, who presented tooth 47 with an extensive mesio-occlusal molten metal restoration, and vestibular fistula at the height of the root bifurcation. Endodontic treatments were performed on the radial thread of the distal root, curettage of the fistula under local anesthesia, provisional restoration and drug prescription according to the patient's clinical need. Final considerations: We can conclude on the report that there is a need for dental surgeons to seek greater knowledge about oral changes caused by aging, so that through proven diagnosis they can indicate more conservative treatments on calcified teeth.
\end{abstract}

Keywords: Calcification, Aging, Treatment.

\section{RESUMEN}

Objetivo: El objetivo de este estudio es presentar un reporte de caso clínico en un paciente anciano atendido en la Clínica de Odontogeriatría en una Universidad del estado de Pará en la ciudad de Belém, que presentaba calcificación interna del diente 47. Detalle del caso: Este es un caso de calcificación pulpar por envejecimiento, confirmado por radiografía periapical: paciente masculino de 70 años, que presentaba el 47

${ }^{1}$ Centro Universitário do Estado do Pará (CESUPA), Belém - PA. *E-mail: evinhasantiago7@gmail.com 
con una extensa restauración mesioclusal de metal fundido y fístula vestibular a la altura de la bifurcación radicular. Se realizaron tratamientos de endodoncia en la rosca radial de la raíz distal, legrado de la fístula bajo anestesia local, restauración provisional y prescripción de fármacos según la necesidad clínica del paciente. Consideraciones finales: Podemos concluir sobre el informe que existe la necesidad de que los cirujanos dentistas busquen un mayor conocimiento sobre los cambios bucales provocados por el envejecimiento, para que mediante un diagnóstico comprobado puedan indicar tratamientos más conservadores en dientes calcificados.

Palabras clave: Calcificación, Envejecimiento, Tratamiento.

\section{INTRODUÇÃO}

O envelhecimento é um processo que ocorre durante a vida que afeta todo o organismo, resultando em alterações de padrões fisiológicos e anatômicos de um indivíduo em uma relação mútua de fatores sociais, culturais, biológicos e psicológicos. O crescimento da população de idosos no Brasil e no mundo está relacionado a uma melhor expectativa de vida devido ao avanço da medicina e resultado do declínio da fecundidade. Dados da OMS (Organização Mundial da Saúde) revelam que as estimativas para o Brasil, a partir do ano de 2025, irão considerá-lo o sexto país do mundo com o maior número de idosos em sua população (RIBEIRO JPM, 2018; MENDES JLV, et al., 2018).

A medida que a população envelhece, surgem novas exigências voltadas à área da saúde. No Brasil, foi criado o Estatuto do Idoso (Lei 10.741 , de $1^{\circ}$ de Outubro de 2003). O capítulo IV-DO DIREITO À SAÚDE. Art.15 assegura atenção integral à saúde do idoso por intermédio do Sistema Único de Saúde (SUS) (BRASIL, 2003).

Para atender às necessidades da população de idosos, deve-se conhecer as alterações causadas pelo envelhecimento da cavidade oral e ter uma visão integral do paciente a ser tratado. O comprometimento da cavidade bucal pode ser afetado à nível funcional, físico e mental e a um convívio social insatisfatório. A complexidade de tratamentos de pacientes idosos, exige um conhecimento e manejo adequado por parte do cirurgião dentista devido a inúmeras limitações do paciente idoso (COLPO GW, 2009).

De 8 a 11 de setembro de 2001, realizou-se, na cidade de Manaus no estado do Amazonas, a segunda Assembleia de Ensino Odontológico- $2^{\circ} \mathrm{ANEO}$ na qual foram reconhecidas 5 novas especialidades, dentre elas a Odontogeriatria, tendo o Conselho Federal de Odontologia (CFO) emitido a Resolução CFO de $n^{\circ} 22$ de 08.12.2001, reconhecendo- a como tal (ROCHA ES, 2006).

Na Resolução CFO - 25/2002, de 16 de maio de 2002, foram estabelecidas as competências para atuação das novas especialidades, dentre elas a Odontogeriatria. Mas, recentemente, foi aprovada a lei $n^{\circ} 13.466$, de 17/07/2017, a qual assegura prioridade especial para os indivíduos de 80 anos de idade ou mais, em todas as ofertas de serviços (lei da Prioridade Especial) (CONSELHO FEDERAL DE ODONTOLOGIA, 2002).

Essa Lei modifica o Estatuto do Idoso (Lei n 10.741/2003). A razão dessa mudança ocorreu em função do aumento da expectativa de vida no Brasil. Hoje a odontogeriatria tornou-se uma especialidade necessária na odontologia (BRASIL, 2017).

Neste estudo, constata-se que a calcificação pulpar ocorre por tecido mineralizado que podem afetar a camara e ou os condutos radiculares de dentes humanos, o qual poderá levar a dificuldades para a prática odontológica devido a obliteração dos mesmos (MEDEIROS FPG, 2017; ROCHA ES, 2006).

A deposição fisiológica da dentina ocorre durante todo o período de vitalidade do dente. A polpa apresenta alterações graduais com a idade em função das inúmeras quantidades de impactos físicos, químicos e biológicos aos quais os dentes são acometidos ao longo da vida do indivíduo até chegar à senilidade. Com a idade, ocorre um aumento na resistência do tecido pulpar devido à redução progressiva de nervos e vasos sanguíneos da polpa que sofre resistência a ação de enzimas proteolíticas e hialoronizidase que podem alterar o colágeno e proteoglicanos da polpa e a morte dos túbulos dentinários onde ocorrem os processos odontoblasticos, resultando no aumento da dentina peritubolar e a esclerose dentinária (JESUS HAF, 2014). 
A obliteração parcial ou total da cavidade pulpar por tecido mineralizado representa um desafio para o clínico no âmbito de localizar e trabalhar os canais radiculares (SANTOS CJA, et al., 2019; COLPO GW, 2009).

As calcificações pulpares são frequentemente achadas despretensiosamente em radiografias, sejam Interproximais e ou panorâmicas. Na maioria das vezes as calcificações pulpares são assintomáticas e a sua localização pode ser coronária e apical, na Camara pulpar e canais radiculares e está fortemente associada ao aumento da idade. Nos casos de necrose pulpar, o tratamento endodôntico deve ser realizado, porém a calcificação pulpar impõe dificuldades, podendo resultar em perfurações coronárias e/ou radiculares afetando negativamente o prognóstico do tratamento (SANTOS CJA, et al., 2019; ROCHA ES, 2006).

Observou-se nos estudos que os dentes mais afetados por calcificações pulpares são os molares com prevalência maior em relação aos pré-molares e incisivos que podem ter uma associação de maior disposição a fatores de agressão (MEDEIROS FPG, 2017; SANTOS AAF, 2015; DA SILVA EJNL, et al., 2017; ANTES $\mathrm{TH}, 2019)$.

A disciplina de Odontogeriatria estuda as manifestações do envelhecimento dos órgãos duros e moles da cavidade oral de forma teórica e prática. Nos casos de calcificação pulpar para se obter um bom tratamento é necessário um correto diagnóstico através da anamnese, exame clínicos, radiografias extra bucais ou periapicais, testes de vitalidade e percussão. Diante disso, o objetivo deste trabalho é evidenciar o sucesso do tratamento realizado no dente o qual apresentava complicações ocasionadas pela calcificação interna fisiológica da polpa e do conduto radicular da raiz distal em paciente idoso.

\section{DETALHAMENTO DO CASO}

Paciente 70 anos, gênero masculino, o qual apresentava o dente 47 com uma restauração metálica fundida extensa meso-oclusal, e fistula vestibular à altura da bifurcação das raízes. Feita a radiografia periapical, verificou-se a calcificação dos condutos radiculares da raiz mesial e suspeita de necrose do filete radicular da raiz distal. $O$ acesso foi realizado na face oclusal da restauração com muita dificuldade devido à calcificação quase completa da câmara pulpar. Consegue-se iniciar a penetração do conduto distal com a Lima tipo Kerr $\mathrm{n}^{\circ} 8$ e realizar a remoção do filete radicular necrosado, curativo com formocresol na Camara pulpar e proteção com IRM. Em seguida, realizou-se a curetagem da fistula sob anestesia local com Citanest (prilocaina +felipressina a $3 \%$ ) meio tubete e a prescrição de amoxicilina 500mg, 1 comprimido de $8 \mathrm{em} 8$ horas, durante 7 dias (Figura 1).

Na sessão seguinte, verificou -se que a fístula havia cicatrizado. Foi realizada a instrumentação do conduto distal com limas tipo Kerr de $n^{\circ} \mathrm{s} 8,10$ e 15. Não houve mais condições de prosseguir devido à dureza da dentina interna da raiz e feita a obturação do conduto com cimento endodôntico Endofill e um cone de gutapercha $n^{\circ} 15$. A perfuração feita na face oclusal da restauração metálica recebeu restauração provisória com cimento de zinco à espera de planejamento da restauração definitiva. $O$ dente permaneceu na cavidade oral do paciente pelo restante de sua vida (7 anos), sem recidiva do processo inflamatório.

Figura 1 - Raio-x Dente 47 calcificação dos condutos radiculares da raiz mesial e suspeita de necrose do filete radicular da raiz distal.

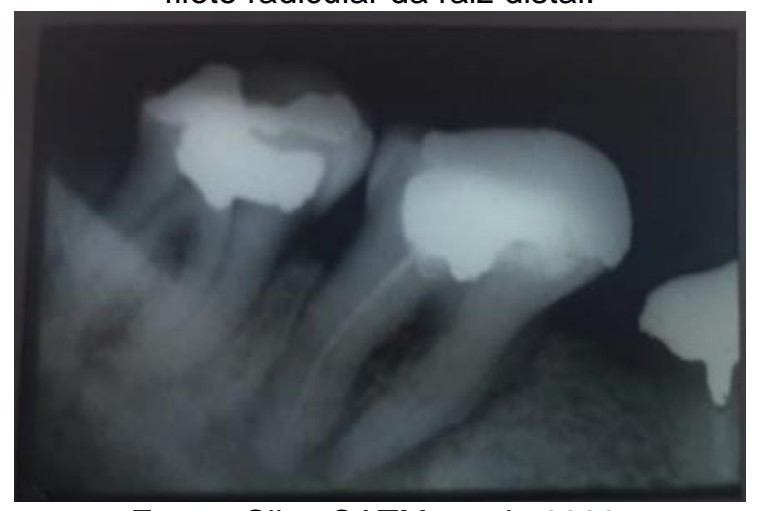

Fonte: Silva CATM, et al., 2020. 


\section{DISCUSSÃO}

A importância do conhecimento da Odontogeriatria para o profissional de odontologia deve ir além do conhecimento sobres os aspectos das particularidades acometidas na cavidade bucal do idoso. é necessário também o conhecimento humano integral do ponto de vista físico, social e intelectual para um bom manejo com responsabilidade para oferecer um atendimento mais humanizado e de qualidade, visto que está faixa etária é constituída de pessoas heterogêneas (NÚÑEZ MDR, et al., 2017).

As mudanças fisiológicas relacionadas a idade nos dentes devem ser cuidadosamente distinguidas de alterações patológicas, especialmente quando induzem dor e um efeito na qualidade de vida relacionada à saúde do paciente geriátrico (CARVALHO TS, 2017).

As mudanças no complexo dentino pulpar por envelhecimento ocorrem inicialmente quando a dentina se torna mais esclerotica e portanto menos elástica. Com a mineralização da dentina peritubolar de forma crescente podem ocorrer resultados de obliteração completa dos tubulos dentinários. Os canais radiculares sofrem um estreitamento contínuo porém a obliteração total é rara. Outra característica de polpas de idosos é a diminuição no número e tamanho das células, diminuição no número de vasos sanguíneos, associado a um aumento de fibras colágenas, ocorre também entre as alterações uma redução de fibras nervosas. Portanto há uma incidência maior de calcificação pulpar em dentes de idosos (RESENDE MR, 2017).

O conhecimento das modificações fisiológicas do envelhecimento sobre saúde oral do idoso deve ser visto pelo cirurgião dentista com a possibilidade de novas terapêuticas e métodos de prevenção, uma vez que o paciente geriátrico possui alterações fisiológicas que levam a condições patológicas próprias do Envelhecimento, sendo necessário ter tratamento diferenciado da população em geral (SANTOS CJA, et al., 2019).

Os pacientes idosos temem tratamentos longos, sofridos e ou onerosos para o tratamento conservador, ocorrendo assim a transição para o edentulismo, por outro lado a indicação da extração dos dentes tem como consequência processo inflamatório, o qual pode ocorrer processos patológicos, prejudicando a função do sistema estomatognático e tornando não praticável o tratamento conservador (CARDOSO MCAF, 2010).

As extrações dos dentes são vistas como a solução mais prática e econômica para os idosos, porque os problemas bucais vêm desde a infância e tornam-se cada vez mais complexos e dispendiosos nessa faixa etária. Atualmente a utilização dos serviços odontológicos pelos pacientes idosos está mudando devido à manutenção dos dentes. Por essa razão deve haver conscientização por parte dos cirurgiões dentistas e prioridade na odontologia, evitar as extrações totais pois a manutenção de uma capacidade mastigatória natural, ainda que limitada pela maioria dos idosos, trata-se de um objetivo importante a ser alcançado por serviços públicos e privados do país (ROCHA ES, 2006).

Há controvérsias na literatura sobre a conduta do tratamento em relação aos dentes com calcificação pulpar. Deve-se usar bom senso assim como o conhecimento científico para decisão de tratamento endodontico ou a exodontia de um elemento dental em pacientes idosos (MCCABE PS; DUMMER, PMH 2012; SANTOS MBF, et al., 2013).

A associação Americana de Endodontia aponta a terapia endodôntica em canais radiculares calcificados como alto nível de dificuldades. E os dentes com obliteração pulpar deverão ser acompanhados e somente diante de sinais e sintomas de doença periapical deverá ser indicado o tratamento endodôntico (SANTOS CJA, et al., 2019; COLPO GW, 2009).

De acordo com o relato de caso mencionado neste estudo, o tratamento endodôntico pode trazer várias dificuldades, segundo expõe a literatura quando se trata de dentes calcificados devido à dureza da dentina $\mathrm{e}$ na tentativa de localizar os canais radiculares, podendo ocorrer erros nos procedimentos, como perfurações, fraturas de instrumentos e desvios do trajeto do canal (SANTOS CJA, et al., 2019; COLPO GW, 2009).

Deve-se buscar o diagnóstico preciso para se obter os melhores resultados de tratamento para cada situação clínica. As calcificações ganham mais relevância clínica quando de necessidade de terapia endodôntica dos dentes afetados. É necessário estudo pela busca por tratamentos de proservação, escolher técnicas conservadoras e menos onerosas em dentes nessas condições clínicas, uma vez que a literatura afirma que os achados de necrose em dentes calcificados é uma condição rara (NOGUEIRA SP, 2014). 
Os tratamentos endodônticos não cirúrgicos são pouco mencionados na literatura, pois existe muita discussão dos autores a respeito da conduta quanto ao tratamento dos dentes com calcificação pulpar. Alguns indicam o tratamento profilático através do tratamento endodôntico para evitar futuros problemas com infecções periapicais. Outros defendem a proservação do elemento dentário com observação clínica e radiografias. A taxa de falha no tratamento Endodôntico não cirúrgico de canais calcificados está entre 10 a 19\% sendo também aconselhada a examinação clínica e radiografica periódica (CAMPOS MBT, 2016).

Em relação ao caso apresentado o mesmo foi bem sucedido pois apesar das implicações clínicas, envolvendo tratamento endodôntico em um dos filetes da raiz distal e curetagem em uma fistula na região vestibular, o paciente obteve acompanhamento clínico e radiográfico, não ocorrendo nenhum processo inflamatório posterior ao tratamento. Portanto, fica evidente que procedimentos menos invasivos em dentes com calcificação interna, por ser um processo natural decorrente do envelhecimento fisiológico, permite aos pacientes receberem tratamentos com menos traumas e mais confortáveis.

O cirurgião dentista precisa ter conhecimento sobre o processo de envelhecimento que acomete a cavidade oral dos pacientes geriátricos, pois as calcificações pulpares são achadas comuns em pacientes acima de 60 anos ou mais. Atualmente, existe uma busca cada vez maior por tratamentos odontológicos por pacientes idosos, o que comprova a evidência do crescimento dessa população, qual busca manter os cuidados da cavidade oral por uma necessidade funcional e estética. Por essa razão, há um maior desafio em adequar protocolos menos invasivos e criar novas técnicas de tratamentos para atender a demanda desse público específico de pacientes e proporcionar cada dia mais a melhora na qualidade de vida deles.

Podemos concluir de positivo, sobre o relato, que há uma necessidade dos profissionais especialistas em dentística e em endodontia, buscarem maior interação entre si e mais conhecimento sobre as alterações fisiológicas do envelhecimento para que possam diagnosticar e indicar o tratamento endodôntico parcial ou total de um conduto calcificado ou apenas o tratamento restaurador, evitando a exodontia, buscando manter por mais tempo o dente calcificado na cavidade oral.

\section{REFERÊNCIAS}

1. ANTES TH. Tratamento endodôntico de dente com mineralização pulpar pós trauma: relato de um caso clínico. Monografia. (Especialização em Endodontia). Curso de Especialização em Endodontia. Universidade Federal do Rio Grande do Sul, Porto Alegre, 2019; p. 30.

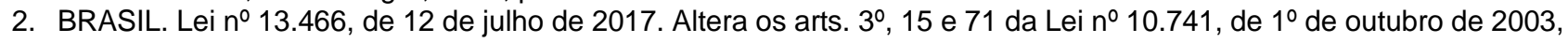
que dispõe sobre o Estatuto do Idoso e dá providências.

3. CAMPOS MBT. Canais calcificados: abordagem em Endodontia. Dissertação. (Mestrado em Medicina Dentária). Faculdade de Ciências da Saúde. Universidade Fernando Pessoa, Porto, 2016; 58p.

4. CARDOSO MCAF, BUJES RV. A saúde bucal e as funções da mastigação e deglutição nos idosos. Estudos Interdisciplinares sobre o Envelhecimento. 2010; 15(1): 53-67.

5. CARVALHO TS, LUSSI A. Age-related morphological, histological and functional changes in teeth. J Oral Rehabil. 2017; 44(4):291-298.

6. CHAGAS AM, ROCHA ED. Aspectos fisiológicos do envelhecimento e contribuição da Odontologia na saúde do idoso. Revista Brasileira de Odontologia. 2012; 69 (1):94-96.

7. COLPO GW. O envelhecimento e suas repercussões na endodontia. Monografia. (Especialização em Endodontia). Curso de Especialização em Endodontia. Universidade Federal do Rio Grande do Sul, Porto Alegre, 2009; 54p.

8. CONSELHO FEDERAL DE ODONTOLOGIA. Resolução CFO25/2002. Diário Oficial da União, Brasília, 25 de maio 2002.

9. DA SILVA EJNL, et al. Assessing pulp stones by cone-beam computed tomography. Clin Oral Investig. 2017; 21(7):2327-2333.

10. DA SILVA MRRR, et al. Microscópio operatório na Endodontia. Research, Society and Development, 2020; 9(8): e981986858-e981986858.

11. ESTATUTO DO IDOSO - Lei $n^{\circ} 10.741$, de $1^{\circ}$ de Outubro de 2003, CAPITULO IV: DO DIREITO À SAÚDE, Art. 15, pág. 11 a 13, publicado no Diário Oficial da União em 03.10.2003 - COORDENAÇÃO ESTADUAL SAUDE DO IDOSO/ Secretaria de Estado de Saúde Pública do Pará.

12. JESUS HAF. Deposição de tecido mineralizado na cavidade pulpar Monografia (Especialização em Endodontia). Faculdade de Odontologia. Universidade Federal de Minas Gerais, Belo Horizonte, 2014; 23p.

13. LIMA DDC. Técnica de remoção de dentina na entrada de canais calcificados de molares. Monografia (Graduação em Odontologia). Universidade Federal de Uberlândia, 2018; 17p.

14. MARQUES IV, et al. Perfuração radicular lateral em um dente com calcificação pulpar: um relato de caso. Archives of Health Investigation, 2018; 7(4): 143-146. 
15. MCCABE PS e DUMMER PMH. Obliteração do canal pulpar: um diagnóstico endodôntico e desafio ao tratamento. Revista endodôntica internacional. 2012; 45(2):177-197.

16. MEDEIROS FBG. Calcificações pulpares-características clínicas, imagenológicas e morfológicas: revisão sistemática. Monografia (Graduação em Odontologia). Centro de Ciências da Saúde. Universidade Federal do Rio Grande do Norte. Natal, 2017; 37p.

17. MENDES JLV, et al. O aumento da população idosa no Brasil e o envelhecimento nas últimas décadas: Uma revisão da literatura. REMAS-Revista Educação, Meio Ambiente e Saúde, 2018; 8(1):13-26.

18. NOGUEIRA SP. Tratamento de dentes com mineralizações e suas implicações: uma revisão de relatos de casos clínicos. Monografia. (Especialização em Endodontia). Curso de Especialização em Endodontia. Universidade Federal do Rio Grande do Sul, Porto Alegre, 2014; 39p.

19. NÚÑEZ MDR, et al. Geriatric dentistry teaching and the curricular guidelines in dental schools in South American countries. Revista Brasileira de Geriatria e Gerontologia. 2017; 20(6):826-835.

20. RESENDE MR. Odontologia na terceira idade. Monografia. (Especialização em Atenção Básica da Família). Curso de Especialização em Atenção Básica da Família. Universidade Federal de Alfenas, Uberaba, 2014; 24p.

21. RIBEIRO JPM. Envelhecimento pulpar em dentes jovens. Dissertação (Mestrado em Medicina Dentária). Instituto Superior de Ciências da Saúde Egas Moniz. Mestrado Integrado em Medicina Dentária. Almada, 2018; 61p.

22. ROCHA ES. Conhecimento e atitudes dos cirurgiões-dentistas de Belo Horizonte em relação ao idoso. Dissertação (Mestrado em Odontologia). Faculdade de Odontologia. Universidade Federal de Minas Gerais, Belo Horizonte, 2006; $114 p$.

23. ROSA L, et al. Odontogeriatria-a saúde bucal na terceira idade. Revista da Faculdade de Odontologia-UPF. 2008; 13(2): 82-86.

24. SANTOS AAF. Sensibilidade pulpar em pacientes geriátricos. Dissertação (Mestrado em Medicina Dentária). Instituto Superior de Ciências da Saúde Egas Moniz. Mestrado Integrado em Medicina Dentária. Almada, 2015; 62p.

25. SANTOS CJA. Calcificação pulpar e implicações clínicas. Monografia (Graduação em Odontologia). Centro de Ciências da Saúde. Universidade Federal de Santa Catarina, Florianópolis, 2019; 40p

26. SANTOS MF, et al. Tratamento endodôntico na terceira idade. RGO. Revista Gaúcha de Odontologia (Online), 2013; 61(0):485-489.

27. TELES AF. Guia virtual endodôntico: uma nova abordagem de tratamento para dentes com calcificação pulpar e periodontite apical. Monografia (Graduação em Odontologia). Faculdade de Odontologia. Centro Universitário do Planalto Central Apparecido dos Santos, Brasília, 2012; $11 \mathrm{p}$.

28. VELOSO ACR. Abordagem clínica a canais radiculares calcificados e/ou atresiados. Dissertação. (Mestrado em Medicina Dentária). Faculdade de Ciências da Saúde. Universidade Fernando Pessoa, Porto, 2017; 39p. 